Boise State University

ScholarWorks

Kinesiology Faculty Publications and Presentations

Department of Kinesiology

$4-1-2012$

\title{
Determining Intensity Levels of Selected Wii Fit Activities in College Aged Individuals
}

Joshua D. Griese

U.S. Air Force

Yong Gao

Boise State University

Lynda Ransdell

Boise State University

Shawn R. Simonson

Boise State University

This is an electronic version of an article published in Measurement in Physical Education and Exercise Science, 16(2), 135-150. Measurement in Physical

Education and Exercise Science is available online at: www.tandfonline.com. DOI: 10.1080/1091367X.2012.665268 


\title{
Determining Intensity Levels of Selected Wii Fit Activities in College Aged Individuals
}

\author{
Joshua D. Griese \\ 92d Force Support Squadron, U.S. Air Force \\ Yong Gao, Lynda Ransdell, and Shawn Simonson \\ Boise State University
}

\begin{abstract}
To determine intensity of Nintendo Wii Fit games using indirect calorimetry. Twenty-five college students completed Wii Fit activity sessions at two difficulty levels within aerobics, strength, and yoga categories. Resting metabolic rate and exercise $\dot{V} \mathrm{O}_{2}$ were measured and metabolic equivalents (METs) were calculated. Measured METs and calculated METs ranged from $2.30 \pm .42$ and $2.44 \pm .38$ for Yoga to $5.73 \pm 1.36$ and $6.04 \pm 1.09$ for aerobics, respectively. All selected Wii Fit activity intensities were significantly higher than resting, $\mathrm{p}<$ .001 ; aerobic and strength activities met the moderate intensity threshold (3 METs, $\mathrm{p}<.001$ ), yoga activities did not $(\mathrm{p}<.005)$; aerobic and yoga activities of medium difficulty were more intense than the corresponding easy-rated activities $(\mathrm{p}<.005)$. There were no statistical differences between measured and calculated METs. Wii Fit has potential as an effective tool for helping college-age individuals increase their PA.
\end{abstract}

Keywords: metabolic equivalent; energy expenditure; exercise-themed video game; physical activity intervention; physical activity prescription

\section{Introduction}

Despite the well-documented health benefits of physical activity (PA), many Americans are still living sedentary or less active lifestyles (Bennett, Wolin, Puleo, Masse, \& Atienza, 2009; Hawkins et al., 2009; King, Mainous, Carnemolla, \& Everett, 2009). According to the Health People 2010 Database (Center for Disease Control and Prevention [CDC], 2010), more than one fourth of US adults did not participate in any leisure time PA in 2008; and only one third participated in the recommended level of PA. Over the years, many intervention programs have been developed to increase PA participation, yet the success seems to be limited. The fact that the prevalence of Americans who are inactive has not changed much in the past decades (CDC, 2010; Pate et al., 1995; U.S. Department of Health and Human Services [USDHHS], 2008) indicates a clear need for more effective PA interventions.

Technology is often blamed for decreases in individuals PA levels. However, the continued development of interactive technology has facilitated some incredible advancement in the PA promotion field (Zhu, 2008). Since a majority of people in the U.S. now have access to different technological advances through personally owned, work, or public use equipment, the use of technology has significant potential for increasing PA on a large-scale basis (Nigg, 2003; Zhu, 2008). Longitudinal studies have found that the use of technology could theoretically provide greater access to PA interventions and may be the most efficient technique for promoting and implementing future interventions (Hillier, 2008). Research has already begun examining the use of technology such as active video gaming systems as a PA promotion tool (Graves, Ridgers, \& Stratton, 2008; Sell, Lillie, \& Taylor, 2008; Tan, Aziz, Chua, \& Teh, 2002).

In the past, active video game play was found mostly in arcades due to the large size of game consoles such as Dance Dance Revolution. Therefore, access to these games was limited. The recent evolution of home based video game consoles by Nintendo revolutionized home video game play by incorporating player movement into video game control (Nintendo of America Inc., 2009). The improvement of video game consoles to home based systems has significantly increased the number of people who can now access this technology (Williams, Yee, \& Caplan, 2008). The Nintendo Wii system uses unique motion sensing technology to determine the location of handheld 
controllers, called the Wii remote and Wii nunchuk, while lower body movement is sensed from the Wii Balance Board placed on the floor. The ability of game designers to incorporate movements for successful game play has allowed for the creation of exercise-themed games.

One such exercise-themed game is "Nintendo Wii Fit", which was the first fitness game released and has remained the most popular in the U.S. since its inception (VGCharts Network, 2009). Wii Fit uses the Wii remote, nunchuk, and Balance Board for the completion of Wii Fit activities that require both upper and lower extremity movement during game play (Nintendo of America Inc., 2009). Wii Fit includes over 40 activities in four different components of fitness (Nintendo of America Inc., 2009): aerobics, strength, yoga, and balance (See Table 1 for a full list of activities). Aerobic and balance game activities incorporate on screen mini games such as snowboarding or boxing to complete these activities; strength and yoga activities are accomplished by following an on screen trainer who demonstrates proper technique. The Wii Fit exercise game also offers three difficulty levels for each fitness component, categorized as easy, medium, and hard.

Given the popularity of Nintendo Wii, the use of this game system to promote PA seems promising. The popularity of the Wii implies greater access to exercise-themed games and allows interventions to be developed that could reach larger populations. Initial studies have indicated that energy expended while playing the Nintendo Wii is greater than traditional video game play and resting energy expenditure (Graves et al., 2008; Graves, Stratton, Ridgers, \& Cable, 2008; Mellecker \& McManus, 2008).

Knowing the energy expenditure of an activity is important for effective PA prescriptions. An essential component used to calculate the energy cost of a specific activity is the activity's intensity level. Research has shown that training adaptations occur due to a combination of three factors: frequency, intensity, and duration of PA. Current PA recommendations state that the frequency and duration of PA recommendations change based on an activity's intensity level (USDHHS, 2008). For example, for aerobic exercise, a person can manipulate exercise intensity and frequency, and exercise five times a week at moderate intensity for 30 minutes or three times a week at vigorous intensity for 25 minutes. Both moderate and vigorous intensity exercises result in benefits, but a person can choose to exercise more frequently at a lower intensity or less frequently at a higher intensity for positive health adaptations. One measure of intensity level are metabolic equivalent (MET) values, which are numeric values equivalent to the number of times above resting energy expenditure that an activity requires. One MET is equal to the amount of energy that the body expends at rest and is fixed at $3.5 \mathrm{~mL} / \mathrm{kg} / \mathrm{min}$ (Aisworth et al., 2000). PA professionals use METs to quantify intensity level of an activity. For example, moderate aerobic activities are classified as expending 3.0 to 5.9 METs and vigorous intensity aerobic activities require expending 6.0 METs or more (Ainsworth et al., 2000; USDHHS, 2008). The PA recommendations suggest that individuals participate in at least 150 minutes of moderate intensity or 75 minutes of vigorous intensity aerobic activity each week (USDHHS, 2008).

Compendia of PA intensities have been developed to help differentiate intensity levels between everyday activities and improve exercise prescription (Ainsworth et al., 2000). The most recent update of the Compendium (Ainsworth et al., 2011) has included METs of Wii activities. However, the intensity levels of Wii game activities have not been thoroughly researched. For example, Wii activities were classified into three categories (i.e., low effort, moderate effort and vigorous effort) in the updated Compendium with one universal MET value for each category (Ainsworth et al., 2011). It is not clear whether the aforementioned three categories are corresponding to the three difficulty levels (i.e., easy, medium difficulty and hard) defined by Wii Fit game system. Wii game players may be more familiar with the difficulty levels than the effort levels. Therefore, to give users more information about the calories they might expend while playing Wii, it would be helpful to provide them with information about the METs of Wii activities at different difficulty levels rather than using effort levels.

In addition, research has shown that the standard resting metabolic rate (RMR) of $3.5 \mathrm{~mL} / \mathrm{kg} / \mathrm{min}$ used to calculate METs in the PA Compendium is significantly higher than the measured, individual's own RMR (e.g., Byrne, Hills, Hunter, Weinsier, \& Schutz, 2005). This means METs computed from the standard RMR (the calculated METs) would be lower than METs calculated from measured RMR (the measured METs). Thus, energy cost of an activity may be underestimated if the calculated METs were used. A correction factor has been recommended to adjust the calculated MET values in such situations (Byrne et al., 2005). However, it is not clear whether an adjustment is needed for estimated versus calculated METs of Wii activities. Therefore, it is important to determine if a difference exists between the measured METs and the calculated METs of Wii Fit activities so that the energy expenditure while playing Wii games can be accurately estimated. 
Given the potential for the Nintendo Wii to become a valuable PA intervention tool, the need to better understand the energy expenditure and intensity aspects of active video gaming has arisen. The purpose of this study was to provide detailed information regarding intensity levels of playing several selected Nintendo Wii Fit games using indirect calorimetry. More specifically, this study was designed to determine: (a) if the METs of selected Wii Fit activities were greater than the resting MET; (b) if the selected Wii Fit activities met the moderate PA intensity threshold; (c) if there was a difference in METs of selected Wii activities between different difficulty levels; and (d) if there was a difference between the measured METs and the calculated METs in Wii Fit activities.

\section{Methods}

\section{Participants}

Twenty-five college students (20 females, 5 males) aged 18 to 29 yrs who had used the Wii less than 10 previous times were recruited from a university campus through e-mails, posted announcements, and word of mouth. Because participants' RMR and working metabolic rates were measured and used in this study to determine activity intensities for the selected Wii Fit activities, the factors that might affect RMR were controlled. Participants were excluded if they had one of the following conditions: outside the age range of 18-29 years, taking any medications that would affect metabolic rate (e.g., beta-blockers or diet pills), or having previous self-reported injuries that might limit mobility or their ability to complete the selected Wii game activities (Pollock et al., 1998). In addition, individuals with previous seizure episodes or epilepsy diagnosis or with body weight exceeding $135.87 \mathrm{~kg}$ (300 pounds) were excluded from the study to comply with the manufacturer recommendations for Wii game play (Nintendo of America Inc., 2009). This study was approved by the Institutional Review Board and a written informed consent was obtained from all participants prior to data collection.

\section{Selected Wii Game Activities}

The "Nintendo Wii Fit" includes over 40 game activities in four different components of fitness: aerobics, strength, flexibility, and balance. It also offers three difficulty levels for each fitness component, ranging from easy to hard. The intent of the current study was to determine the intensity levels of selected aerobics, strength, and yoga flexibility activities at easy and medium difficulty levels. Aerobic activities consisted of running-type activities and were the Basic Run Short (easy) and the Basic Run Long (medium difficulty) from the game. Lunges (easy) and Single Leg Extensions (medium difficulty) were the strength activities. The yoga activities included the Warrior Pose and the Chair Pose. Table 2 presents a full description of these activities.

\section{Measures}

Participants completed a consent form, a demographic and general health questionnaire (e.g. age, gender, Wii usage, current medications), the PA readiness questionnaire (PAR-Q; Thomas, Reading, \& Shephard, 1992), and the International PA Questionnaire (IPA-Q; Craig et al., 2003; Fogelholm et al., 2006). The PAR-Q is a standard screening tool that is recommended by the American College of Sports Medicine (ACSM) for exercise testing and prescription (Thompson, Gordon, \& Pescatello, 2010). It was used in this study to determine if a participant was unlikely to experience adverse cardiac and orthopedic events during participation in the selected Wii activities. The IPA-Q has been validated for PA assessment in 15-69 year olds and was used to determine the participants' selfreported PA level in this study. The IPA-Q measured duration and frequency that participants engaged in moderate and vigorous PA in the domains of work, transportation, household and leisure during the last seven days. The IPA$\mathrm{Q}$ response scores were expressed as energy expenditure in the format of MET-minutes/week, which was then used to classify participants into high, moderate, or low activity categories according to the standard IPA-Q scoring protocol (Craig et al., 2003).

Height was measured to the nearest half centimeter using a stadiometer (Seca, Chino, CA). Body mass was measured using a BWB-800S Digital Scale (Tanita Corporation of America, Arlington Heights, IL). Skinfold measurements (rounded to the nearest tenth of a millimeter) were taken using a Harpenden skinfold caliper (Baty International, Sussex, England) on gender-specific sites: Chest, abdomen and thigh skinfolds for males, and triceps, anterior suprailliac and thigh skinfolds for females (Jackson \& Pollock, 1978; Jackson \& Pollock, 1980). The validated Jackson-Pollock 3-site body density equations and the Siri equation were used to calculate percent body fat (Jackson \& Pollock, 1978; Jackson \& Pollock, 1980; Jackson \& Pollock, 1985; Wilmore, 1978). 
Heart rate was measured with a Polar heart rate monitor (Polar Electro, Inc., Kempele Finland). The rate of relative oxygen uptake $\left(\dot{V} \mathrm{O}_{2} ; \mathrm{mL} / \mathrm{kg} / \mathrm{min}\right)$ was measured breath to breath using a TrueMax 2400 Metabolic Cart (ParvoMedics, Salt Lake City, Utah), a valid device for measuring gas consumption and production during exercise and at rest (Bassett et al., 2001; Crouter, Antczak, Hudak, DellaValle \& Haas, 2006; Hodges, Brodie, \& Bromley, 2005). The metabolic cart was calibrated prior to each data collection session following the manufacturer's protocol. Each Wii game activity was played for a minimum of three minutes to ensure that a steady state was reached. Steady state was determined by measured heart rates in consecutive minutes with less than five beats per minute variability. The $\dot{V} \mathrm{O}_{2}$ for each Wii game activity was determined as the average of the final two minutes of the activity. The measured Metabolic Equivalents (MET) values were calculated by dividing $\dot{\mathrm{V}} \mathrm{O}_{2}$ by each participant's own RMR, and the calculated METs were computed by dividing $\dot{V} \mathrm{O}_{2}$ by standardized RMR of $3.5 \mathrm{~mL} / \mathrm{kg} / \mathrm{min}$.

\section{Procedure}

Each participant was required to come to the research lab for a total of four visits. During Visit one, participants were asked to complete a consent form, the demographic and general health questionnaire, the PAR-Q, and the IPAQ. These questionnaires were reviewed and all participants passed the screening for study enrollment. Therefore, they were scheduled for another three visits. Participants were instructed to dress for activity, fast for 12 hours, and refrain from vigorous PA 24 hours prior to arrival for Visit two.

During Visit two, after measuring height, weight, and completing skinfold measurements, participants sat in a comfortable position and relaxed for 20 minutes. Then their resting heart rate (RHR) was measured using the heart rate monitor, followed by the measurement of RMR with the metabolic cart for 10 minutes. Participants were then given at least 30 minutes to play and become familiar with the Wii system. Participants were reminded to refrain from high intensity PA for 24 hours prior to the Wii game play sessions (Visits 3 and 4).

During Visits 3 and 4, $\dot{\mathrm{VO}}{ }_{2}$ was measured using the metabolic cart during six selected Wii game activities, each lasting at least three minutes (ranging 3 to 7 minutes). The Wii strength activities typically ranged from 10 repetitions to 30 repetitions with three levels of repetitions for each activity which can be selected by the user. In this study, the middle repetition number was selected for the two strength activities, which required 20 repetitions from each side of the body. The activities and pace are most similar to what would occur in a circuit style group exercise class using body weight or small dumbbells as resistance. The yoga poses were repeated with each set lasting between 30 to 60 seconds; individuals were required to stress the limits of the range of motion around a specific joint.

The testing order of the six Wii activities was randomly determined by each participant to eliminate the effect of testing order on study results. Each of the six Wii activities was assigned a starting number based on rolling dice. Participants then rolled the dice to determine their starting Wii game activity; the second and third Wii game activities were the next two sequentially numbered activities. The second Wii game play visit continued with the next sequentially numbered activity followed in order by the final two Wii game activities (e.g., if a three was rolled then the first Wii activity of the fourth visit would be activity number six, followed by number one and number two). Table 2 presents a schematic of the order of the activities.

Prior to the start of each Wii activity, a demonstration of that activity was shown to each participant. Additional technique and form instructions were given to ensure the proper completion of each activity. Participants then completed a five-minute warm-up by walking at a self-selected ungraded speed on a treadmill; next, they wore a heart rate monitor and were connected to the metabolic cart for $\dot{\mathrm{VO}}{ }_{2}$ measurement. Participants then completed the Wii game activity protocol according to the randomly determined testing order. Participants were given a rest period in between the Wii activity sets to ensure no effect from the previous activity. The rest period was completed when the participant's heart rate dropped below 120\% of his/her RHR. Upon completion of the Wii game activity protocol, participants cooled-down by walking on a treadmill until heart rate had fallen to within 30 beats of RHR. 


\section{Data Analysis}

SPSS 18.0 (Chicago, IL) was used for all statistical analyses. Descriptive statistics were conducted for age, gender, height, weight, body mass index (BMI), body composition, Wii familiarity, IPA-Q scores, the resting physiological parameters such as RHR and RMR, and $\dot{V} \mathrm{O}_{2}$ and METs for each selected Wii game activity. One sample t-tests were used to determine if the METs of each Wii game activity were significantly higher than 1 MET (the standard resting MET value) and if intensities of playing the Nintendo Wii Fit games met or exceeded 3 METs, the moderate PA intensity level recommended by ACSM (Pate et al., 1995; USDHHS, 2008). A set of repeated measures ANOVAs was used to determine if Wii game activities at the medium difficulty level had significantly higher MET values compared to the easy Wii game activities, and whether there was a significant difference for each activity between the measured METs and the calculated METs. Due to the fact that both PA levels and body composition affect RMR (Byrne et al., 2005; Hoeger \& Hoeger, 2008) -- which can ultimately affect the calculation of METs -self reported PA scores from IPA-Q and percent body fat were used as covariates when analyzing the MET differences. Statistical significance for MET value comparisons in this study was set at .005 to account for the potential inflation of type I error due to multiple comparisons, and the fact that the dependent variables (i.e., the MET values of these activities) were likely correlated. In addition, Partial Eta Squared (a measure of effect size) and statistical power were reported when relevant. An effect size with Partial Eta Squared of .04 is typically considered small, and .25 and .64 are considered medium and large, respectively (Cohen, 1988; Ferguson, 2009; Pierce, Block \& Aguinis, 2004).

\section{Results}

Table 3 reports the demographic, physiological, and physical activity characteristics of the sample overall and by gender. The sample consisted of 20 female and 5 male participants (age: $22 \pm 2$ years; mean $\pm S D$ ) who were physically active (IPA-Q score $=4482 \pm 2822 \mathrm{MET}$-min/week) and fit $\left(\mathrm{BMI}=23.31 \pm 3.01 \mathrm{~kg} / \mathrm{m}^{2}\right.$, body fat $=17.92$ $\pm 6.22 \%$, and lean body mass $=54.36 \pm 9.97 \mathrm{~kg})$. Male participants were significantly taller $(p=.004)$ and heavier $(p<.001)$ than female participants. Male participants also had significantly higher BMI $(p=.007)$ and lean body mass $(p<.001)$ than female participants, but there were no gender difference in percent body fat $(p=.18)$. No statistically significant gender differences in the IPA-Q scores (in the format of MET-min/week) and RMR were revealed, $t(23)=1.80, p=.09$ and $t(23)=.25, p=.80$, respectively.

Table 4 presents average $\dot{\mathrm{V}} \mathrm{O}_{2}$ and measured and calculated MET values for the participants. Average $\dot{\mathrm{V}} \mathrm{O}_{2}$ ranged from $8.53 \pm 1.33 \mathrm{~mL} / \mathrm{kg} / \mathrm{min}$ for the Yoga Warrior activity to $20.98 \pm 3.65 \mathrm{~mL} / \mathrm{kg} / \mathrm{min}$ for the aerobics Basic Run Long activity. Correspondingly, the mean measured MET (activity $\dot{V} \mathrm{O}_{2}$ divided by participant's own RMR) and calculated MET (activity $\dot{V} O_{2}$ divided by a standard RMR of $3.5 \mathrm{~mL} / \mathrm{kg} / \mathrm{min}$ ) ranged from $2.30 \pm .42$ METs and $2.44 \pm .38$ METs for the Yoga Warrior activity to $5.73 \pm 1.36$ METs and $6.04 \pm 1.09$ METs for the Basic Run Long aerobic activity, respectively. The aerobics themed activities had the highest $\dot{V} O_{2}$ and MET values, followed by the Wii strength activities, then the two yoga poses. The MET values of both yoga and strength activities in this study were similar to those listed in the 2011 PA Compendium (2.3 METs for yoga and 3.8 METs for strength activities, respectively). Although aerobic activities had higher METs $(p<.0005)$ in this study compared to the 3.8 METs listed in the Compendium, they are still in the moderate intensity category (Ainsworth et al., 2011).

\section{METs of Selected Wii Activities Compared With Resting MET}

A set of one-sample t-tests was conducted to examine if the intensity levels of selected Wii Fit activities were significantly higher than resting (1 MET). The comparisons were conducted for both the measured and the calculated METs of each Wii Fit activity. The lowest metabolic rate for the selected Wii activities was the Yoga Warrior. The mean measured METs for the Yoga Warrior was $2.30 \pm .42$ METs, which was significantly higher than resting, $t(24)=15.53, p<.001$. The mean calculated MET score for the Yoga Warrior was $2.44 \pm .38$ METs, which was also significantly higher than resting, $t(24)=19.1, p<.001$. Statistical analyses revealed that the measured and the calculated METs of all other activities were significantly higher than resting, with $t$ values ranging from 15.53 for the Yoga Warrior to 23.04 for the aerobics Basic Run Long, $(p<.001)$. 


\section{METs of Selected Wii Activities Compared With Moderate Intensity Threshold}

A set of one-sample t-tests was then conducted to compare the intensity levels of selected Wii Fit activities to the moderate intensity PA threshold of 3 METs as defined by ACSM. Activities that were significantly higher in intensity than moderate activity (3 METs) in both measured METs and calculated METs were the aerobics Basic Run Short and Long activities, with $t(24)=8.15$ for the measured METs and $t(24)=11.25$ for the calculated METs for the Basic Run Short activity, $p<.001$; and $t(24)=10.04$ for the measured METs and $t(24)=13.90$ for the calculated METs for the Basic Run Long activity, $p<.001$.

The measured and calculated MET values for each yoga activity were significantly lower than the moderate PA intensity threshold of 3 METs, with $t(24)=-8.37$ for the measured MET value and $t(24)=-7.45$ for the calculated METs for Yoga Warrior activity, $p<.001$; and $t(24)=-6.82$ for the measured MET value and $t(24)=-3.35$ for the calculated METs for Yoga Chair activity, $p=.003$.

No statistical differences were found between the measured METs of strength activities $(t(24)=1.49, p=.15$ for Lunges and $t(24)=2.49, p=.05$ for Single Leg Extension) and the moderate PA intensity threshold of 3 METs, meaning that lunges and single leg extensions, both strength activities, should be considered moderate intensity. However, the calculated METs were significantly higher for the two strength activities when compared to the threshold of 3 METs, with $t(24)=3.31, p=.003$ for Lunges, and $t(24)=4.08, p<.001$ for Single Leg Extension.

\section{MET Differences between Difficulty Levels}

A set of repeated measures ANOVAs was conducted to determine if the intensities between easy and medium difficulty activities were statistically different. The results indicated that medium difficult Basic Run Long aerobic activity (measured METs $=5.73 \pm 1.36 \mathrm{METs}$ ) was significantly more intense than the easy aerobic activity Basic Run Short (measured METs = $4.98 \pm 1.22$ METs), $F(1,24)=24.95, p<.001$, Partial Eta Squared $=.51$, Power $=$ .99. The significance disappeared when either IPA-Q scores $(p=.02$, Partial Eta Squared $=.22$, Power $=.69)$ that reflected participants' PA levels or percent body fat $(p=.3$, Partial Eta Squared $=.04$, Power $=.17)$ was included as a covariate in data analysis. The similar results were confirmed for the calculated METs between Basic Run Short and Long activities.

There was a statistical difference between the Yoga Warrior MET value intensity (measured METs $=2.30 \pm .42$ METs), and the Yoga Chair pose mean intensity (measured METs = $2.51 \pm .36 \mathrm{METs}), F(1,24)=10.42, p=.004$, Partial Eta Squared $=.30$, Power $=.87$. However, no difference was observed when either IPA-Q scores or percent body fat was included in the analysis, $p=.22$, Partial Eta Squared $=.07$, Power $=.22$ and $p=.30$, Partial Eta Squared $=.05$, Power $=.19$, respectively. Similar patterns regarding the calculated METs differences between Yoga Warrior and Yoga Chair were observed.

There was no statistical difference between MET values for easy and medium difficulty levels for strength activities, $F(1,24)=.90, p=.35$, Partial Eta Squared $=.04$, Power $=.15$. This was true for both the measured and calculated METs, with or without controlling participants’ PA levels or percent body fat.

\section{Differences between Measured METs and Calculated METs}

The measured MET values appeared lower in five (Basic Run Short, Yoga Warrior, Yoga Chair, Lunges, and Single Leg Extension) of the six activities when compared to the calculated MET values; however, the differences were not statistically significant according to the alpha level of .005 set for this study; the F statistic ranged from 4.84 for the easy aerobic activity Basic Run Short to 6.76 for the Yoga Chair activity, $p>.005$. Additionally, effect sizes (Partial Eta Squared <.25) were small and statistical power was lower than .7.

\section{Discussion}

PA is an essential component to a healthy lifestyle and should be a part of everyday life for all individuals. The continued development of PA interventions to combat the lack of PA participation among the U.S. population is of great public heath interest (e.g., Must, Bandini, Tybor, Phillips, Naumova, \& Dietz, 2007). The accessibility of the exercise-themed games such as Nintendo Wii for use at home, in addition to the fun nature of the games provides a 
potential platform for PA intervention. The intensity aspect of these exercise-themed games needs to be thoroughly examined in order to prescribe these games as part of a PA intervention.

\section{Major Findings}

This study was performed to determine the intensity levels of six selected Wii Fit activities through indirect calorimetry. The major findings were that: a) the METs of all six selected Wii Fit activities were significantly higher than resting (1 MET); b) the MET values of the two aerobic activities were significantly higher than the recommended moderate intensity PA threshold of 3 METs, and the MET values of the two strength activities were equal to 3 METs, whereas the intensity level of the two yoga activities was significantly lower than 3 METs. The METs values of yoga and strength activities are comparable to those listed in the 2011 PA Compendium whereas the METs of aerobic activities are significantly higher than the METs listed in the Compendium (Ainsworth et al., 2011); c) the aerobic Basic Run Long and Yoga Chair activities at the medium difficulty level were significantly more intense than the easier aerobic Basic Run Short and Yoga Warrior activities, respectively. However, such differences disappeared when participants' PA levels and/or percent body fat were statistically controlled. No statistical difference in intensity level was found between the two Wii strength activities; and finally d) the measured MET values were lower in five of the six activities when compared to the calculated MET values; however, the differences were not statistically significant based on the alpha level set in this study.

\section{Intensity of Selected Wii Fit Activities}

The finding that all of the selected Wii Fit activities had an intensity level that was significantly higher than resting implies that playing these games burns more calories than participating in common sedentary activities such as watching TV or playing computer games. These results are consistent with other video game research that has found traditional video games require more energy than resting levels and that active video games require significantly more energy than traditional video games (Lanningham-Foster et al., 2006; Mellecker \& McManus, 2008; Sell et al., 2008). PA is defined as any bodily movement that requires energy expenditure above resting levels (Hoeger \& Hoeger, 2008). The six selected Wii Fit activities in this study meet this definition and are likely to represent a basic broad compilation of activities that most individuals would put into a basic exercise routine. Therefore, such results provide useful information to verify the potential for the Nintendo Wii to be used as a PA promotion tool.

The result that the two selected aerobic activities exceeded a moderate intensity level in an active sample of young adults is promising. The moderate intensity PA threshold recommendations are important because they provide the level at which significant health benefits and physical fitness improvements occur. Lower intensity activities, while beneficial, do not provide the same significant health benefits (Haskell et al., 2007; Hoeger \& Hoeger, 2008; Pate et al., 1995; USDHHS, 2008).

The two selected Wii strength activities also met the moderate PA intensity threshold. This information is unique given that very few studies have examined the intensity levels of strength activities in METs. The current recommendations on strength training indicate that strength training should be done twice a week and include all major muscle groups, include 2 to 4 sets per muscle group at intensity equal to $60 \%$ to $70 \%$ of $1 \mathrm{RM}$ for novice and intermediate individuals (Haskell et al., 2007; Pate et al., 1995; USDHHS, 2008). While using percentage of 1 RM to define intensity for strength activities certainly has its advantages for exercise prescription, the caloric expenditure cannot be directly calculated with such percentages. The two movements used in the strength exercises portion of the Wii Fit for this study did not appear intense enough to be classified as strength training in this participant population. However, the MET intensity information obtained for the two Wii Fit strength activities indicated that the participants were expending similar amounts of energy as if they were engaging in moderate aerobic activities while targeting some of the major muscle groups (hamstrings, quads, abdominals; Haskell et al., 2007; Pate et al., 1995; USDHHS, 2008). This information can be used in future exercise and PA prescriptions (i.e., when caloric expenditure information would be beneficial for weight management).

The two selected Wii yoga activities resulted in an intensity level that was significantly lower than the recommended moderate intensity for PA. The yoga poses that were selected in this study were more of the basic poses to ensure that all participants would be able to complete the poses. These poses lasted from 30 to 60 seconds per set and required the individual to stress the limits of the range of motion of the joints and commanded balance. Whether or not the two yoga activities meet the moderate intensity threshold may not be the most important question to answer 
because the moderate intensity threshold is more relevant to aerobic activities. The current ACSM position stand (Garber et al., 2011) recommends flexibility training on at least 2 days per week with a total duration of 60 sec for each of the major muscle groups (10 min total). A well-rounded fitness program should include calorie-burning activities of moderate to vigorous intensity along with strength-building and flexibility activities. Even though the two yoga activities did not reach an intensity level necessary to meet the moderate intensity PA recommendations, participation in these yoga activities can help participants improve their flexibility and balance, which are important components of fitness. In addition, the intensity information of the yoga activities may be useful in a PA prescription when energy expenditure and flexibility and/or balance are both of interest.

\section{Effect of Game Difficulty on Activity Intensity}

Any exercise-themed program would not be complete unless the participant had the ability to increase relative workload to prevent reaching training plateaus (Baechle \& Earle, 2008; Hoeger \& Hoeger, 2008). In other words, easy, medium, and difficult activities should be available so the participant does not get bored and so he or she can adjust activities once they become easier. Therefore, with the Nintendo Wii Fit, it was important to determine if activity difficulty was indeed progressive (resulting in higher energy expenditure) as the participant advanced through levels of difficulty.

The aerobic activity (Basic Run) difficulty levels resulted in a significantly higher intensity level when comparing the long run to the short run. The yoga activities also had a significant difference in intensity between the two difficulty levels. However, the differences in both the running and yoga were not significant after controlling for PA levels and/or body composition. It appears that it was the variations in habitual PA levels and/or body composition that impacted changes of METs in difficulty level. In other words, percent body fat and PA level appear to affect the MET values of Wii Fit activities more than the game itself. The two strength exercises did not demonstrate significant differences in intensity levels between difficulty levels, which indicated that strength activity difficulty development from easy to medium level did not result in a progression of intensity and may not allow for continued adaptation as participants become more fit. However, many of the Wii Fit activities had three options for increasing the difficulty. Theoretically, the intensity would increase for the activities at hard difficulty level compared to those at the easy or medium difficulty level, but this concept needs further development and validation. Future research is needed to compare intensity level differences between hard and easier Wii Fit activities. It is possible that the use of the Wii Fit may be more beneficial only for those at lower fitness levels, but again, that concept needs further development.

\section{Effect of RMR on Activity Intensity}

It is not surprising that the measured and calculated MET values for each selected Wii activity were not statistically different. This is probably because the sample in this study was similar (e.g, young, physically active, and fit) to samples in previous studies used to establish MET values in the PA Compendium (Ainsworth et al., 2000). The measured MET values were directly affected by measured RMR, which may in turn be influenced by PA level and body composition of the participants. With the average measured RMR in this study similar to the standardized RMR of $3.5 \mathrm{~mL} / \mathrm{kg} / \mathrm{min}$ in the MET equation, the resulting intensity would be similar to the intensity calculated from the standardized RMR. Measured METs might have been significantly higher than calculated METs if a sedentary sample was used because individuals who are less active typically have a lower RMR (due to less lean body mass and more fat mass) than individuals who have the same total body mass but are more active (Hoeger \& Hoeger, 2008). Therefore, if individuals using Wii Fit are similar to our sample, calculated METs and energy expenditure can be used for PA recommendations. If individuals are less active and fit, measured energy expenditure might yield more accurate energy expenditure estimations.

\section{Limitations and Suggestions for Future Research}

The results of this study are useful in that the potential of the Nintendo Wii Fit game as a beneficial PA promotion tool is partially shown. Individuals may use moderate Wii Fit activities (aerobic and strength) to maintain fitness and strength or increase their overall caloric expenditure. Individuals may gain flexibility benefits from participating in Wii yoga activities, as flexibility activities are recommended as part of an overall fitness program. Given the activity intensity level is one of the most crucial components of the current PA recommendations, the fact that some of the Wii Fit activities could meet this threshold holds excellent promise for future use of the Wii as a PA promotion tool. 
This is an electronic version of an article published in Measurement in Physical Education and Exercise Science, 16(2), 135-150. Measurement in Physical Education and Exercise Science is available online at: www.tandfonline.com. DOI: $10.1080 / 1091367 X .2012 .665268$

However, this study provides only partial information relative to the potential use of video game technology as a PA promotion tool and it is not without limitations. One limitation was that not all activities were measured, particularly relevant is that none of the hard level activities were measured; therefore, conclusions about the intensity level of each activity in the Wii Fit game cannot be made and intensity differences between difficulty levels are not conclusive. The characteristics (e.g., young, physically active, and fit) of the study sample also limited the ability to generalize the results of this study to a different population until further research can be completed. Furthermore, the study contained a high proportion of women compared to men, which made the gender comparisons in intensities difficult. Finally, the weight threshold of 300 pounds placed on the Nintendo Wii Balance Board minimizes the potential of the Nintendo Wii to be an activity promotion tool in obese individuals over 300 pounds.

Additional research would be beneficial to paint a more detailed picture of the exact capabilities of the Nintendo Wii system and the Nintendo Wii Fit game. For the Wii Fit game itself, further research should be conducted using all activities and larger samples to determine which ones are the most intense and which activities provide little benefit. Exploration of how game experience affects energy expenditure is also desirable. A third area of interest is how changing intensity and activities within the Wii Fit game affect adherence to physical activity. Fourth, research to establish the intensity level of the system in other populations would also be beneficial as the Nintendo Wii is used by children as a physical education alternative (Brooks \& Brooks, 2009; Hellmich, 2010) and in elderly individuals for balance improvement (Brown, Sugarman, \& Burstin, 2009). The determination of the game's ability to improve physical fitness and progress an individual to allow for continued adaptation would establish the ability of the Wii to act as a standalone PA platform.

\section{Conclusions}

The METs of the selected Wii yoga and strength activities determined in this study are comparable to those listed in the Compendium, and some of the selected Wii Fit activities met the moderate PA intensity threshold. Given the popularity of the Nintendo Wii system, the potential benefit of the Nintendo Wii Fit game and/or the Nintendo Wii system to become a satisfactory PA promotion tool is encouraging. The findings of this study will allow for the further advancement of exercise-themed video games to become PA intervention platforms. 


\section{References}

Ainsworth, B. E., Haskell, W. L., Whitt, M. C., Irwin, M. L., Swartz, A. M., Strath, S. J., . . Leon, A. S. (2000). Compendium of physical activity: An update of activity codes and MET intensities. Medicine and Science in Sports and Exercise, 32, S498-S516.

Ainsworth, B. E., Haskell, W. L., Herrmann, S. D., Meckes, N., Bassett Jr. D. R., Tudor-Locke, C., . . Leon, A. S. (2011). 2011 Compendium of Physical Activities: a second update of codes and MET values. Medicine and Science in Sports and Exercise, 43(8), 1575-1581.

Baechle, T. R., \& Earle, R. W. (2008). Essentials of strength training and conditioning. Champaign (IL): Human Kinetics.

Bassett, D. R., Howley, E. T., Thompson, D. L., King, G. A., Strath, S. G., McLaughlin, J. E., \& Parr, B. B. (2001). Validity of inspiratory and expiratory methods of measuring gas exchange with a computerized system. Journal of Applied Physiology, 91(1), 218-224.

Bennett, G. G., Wolin, K. Y., Puleo, E. M., Masse, L. C., \& Atienza, A. A. (2009). Awareness of national physical activity recommendations for health promotion among US adults. Medicine and Science in Sports and Exercise, 41(10), 1849-1855.

Brooks, D., \& Brooks, S. (2009). Teaching wellness through Wii Fit. (2009). Retrieved from http://www.hotchalk.com/mydesk/index.php/editorial/121-classroom-best-practices/567-teaching-wellnessthrough-wii-fit-.

Brown, R., Sugarman, H., \& Burstin, A. (2009). Use of Nintendo Wii Fit for treatment of balance problems in an elderly patient with stroke: A case report. International Journal of Rehabilitation Research, 32(1), S109S110a.

Byrne, N. M., Hills, A. P., Hunter, G. R., Weinsier, R. L. \& Schutz, Y. (2005). Metabolic equivalent: One size does not fit all. Journal of Applied Physiology, 99(3), 1112-1119.

Centers for Disease Control and Prevention (CDC). Data 2010: the health people 2010 database. (2010). Retrieved from http://wonder.cdc.gov/data2010/focus.htm.

Cohen, J. 1988. Statistical power analysis for the behavioral sciences ( $2^{\text {nd }}$ ed.). Hillsdale, NJ: Lawrence Erlbaum Associates.

Craig, C. L., Marshall, A. L., Sjöström, M., Bauman, A. E., Booth, M. L., Ainsworth, B. E., . . Oja, P. (2003). International physical activity questionnaire: 12-country reliability and validity. Medicine and Science in Sports and Exercise, 35, 1381-1395.

Crouter, S. E., Antczak, A., Hudak, J. R., DellaValle, D. M., \& Haas, J. D. (2006). Accuracy and reliability of the ParvoMedics TrueOne 2400 and MedGraphics VO2000 metabolic systems. European Journal of Applied Physiology, 98(2), 139-151.

Ferguson, C. J. (2009). An effect size primer: A guide for clinicians and researchers. Professional Psychology: Research and Practice, 40, 532-538.

Fogelholm, M., Malmberg, J., Suni, J., Santtila, M., Kyröläinen, H., Mäntysaari, M., \& Oja, P. (2006). International physical activity questionnaire: Validity against fitness. Medicine and Science in Sports and Exercise, 38(4), 753-760.

Garber, C. E., Blissmer, B., Deschenes, M. R., Franklin, B. A., Lamonte, M. J., Lee, I. M., . . Swain, D. P. (2011). Quantity and quality of exercise for developing and maintaining cardiorespiratory, musculoskeletal, and neuromotor fitness in apparently healthy adults: Guidance for prescribing exercise. Medicine and Science in Sports and Exercise, 43(7), 1334-1359.

Thompson, W.R, Gordon, N. F., Pescatello, L. S. (Eds.) (2010). ACSM’s guidelines for exercise testing and prescription. Philadelphia: Lippincott Williams \& Wilkins.

Graves, L. E., Ridgers, N. D., \& Stratton, G. (2008). The contribution of upper limb and total body movement to adolescents' energy expenditure whilst playing Nintendo Wii. European Journal of Applied Physiology, 104(4), 617-623.

Graves, L., Stratton, G., Ridgers, N. D., \& Cable, N. T. (2008). Energy expenditure in adolescents playing new generation computer games. British Journal of Sports Medicine, 42(7), 592-594.

Haskell, W. L., Lee, I. M., Pate, R. R., Powell, K. E., Blair, S. N., Franklin, B. A., . . Bauman, A. (2007). Physical activity and public health: Updated recommendation for adults from the American College of Sports Medicine and the American Heart Association. Medicine and Science in Sports and Exercise, 39(8), 14231434.

Hawkins, M. S., Storti, K. L., Richardson, C. R., King, W. C., Strath, S. J., Holleman, R. G., \& Kriska, A. M. (2009). Objectively measured physical activity of USA adults by sex, age, and racial/ethnic groups: a cross- 
sectional study. International Journal of Behavioral Nutrition and Physical Activity. Retrieved from http://www.ncbi.nlm.nih.gov/pmc/articles/PMC2701914/pdf/1479-5868-6-31.pdf.

Hellmich, N. (2010). Video games help schools get kids moving, exercising more. USA Today Retrieved from http://www.usatoday.com/yourlife/fitness/2010-10-11-justdance11_CV_N.htm.

Hillier, A. (2008). Childhood overweight and the built environment: Making technology part of the solution rather than part of the problem. Annals of the American Academy of Political and Social Science, 615(1), 56-82.

Hodges, L. D., Brodie, D. A., \& Bromley, P. D. (2005). Validity and reliability of selected commercially available metabolic analyzer systems. Scandinavian Journal of Medicine and Science in Sports, 15(5), 271-279.

Hoeger, W. K., \& Hoeger, S. A. (2008). Principles and labs for fitness \& wellness. Belmont (CA): Wadsworth Cengage Learning.

Jackson, A. S., \& Pollock, M. L. (1978). Generalized equations for predicting body density of men. British Journal of Nutrition, 40(3), 497-504.

Jackson, A. S., Pollock, M. L., \& Ward, A. (1980). Generalized equations for predicting body density of women. Medicine and Science in Sports and Exercise, 12(3), 175-182.

Jackson, A. S., \& Pollock, M. L. (1985). Practical assessment of body composition. Physician Sportsmed, 13(5), 7690.

King, D. E., Mainous, A. G., Carnemolla, M., \& Everett, C. J. (2009). Adherence to healthy lifestyle habits in US adults, 1988-2006. American Journal of Medicine, 122(6), 528-534.

Lanningham-Foster, L., Jensen, T. B., Foster, R. C., Redmond, A. B., Walker, B. A., Heinz, D., \& Levine, J. A. (2006). Energy expenditure of sedentary screen time compared with active screen time for children. Pediatrics, 118(6), E1831-E1835.

Mellecker, R. R., \& McManus, A. M. (2008). Energy expenditure and cardiovascular responses to seated and active gaming in children. Archives of Pediatrics \& Adolescent Medicine, 162(9), 886-891.

Mhurchu, C. N., Maddison, R., Jiang, Y., Jull, A., Prapavessis, H., \& Rodgers, A. (2008). Couch potatoes to jumping beans: a pilot study of active video games on physical activity and body composition in children. International Journal of Behavioral Nutrition and Physical Activity, 5(8), 1-5. doi:10.1186/1479-5868-5-8

Must, A., Bandini, L. G., Tybor, D. J., Phillips, S. M., Naumova, E. N., \& Dietz, W. H. (2007). Activity, inactivity, and screen time in relation to weight and fatness over adolescence in girls. Obesity, 15(7), 1774-1781.

Nigg, C. (2003). Technology's influence on physical activity and exercise science: The present and the future. Psychology of Sport and Exercise, 4(1): 57-65.

Nintendo of America Inc. Wii Fit at Nintendo. (2009). Retrieved from http://www.nintendo.com/games/detail/hoiNtus4JvIcPtP8LQPyud4Kyy393oep.

Pate, R. R., Pratt, M., Blair, S. N., Haskell, W. L., Macera, C. A., Bouchard, C., . . Wilmore, J. H. (1995). Physical activity and public-health: a recommendation from the centers for disease control and prevention and the American college of sports medicine. Journal of the American Medical Association, 273(5), 402-407.

Pierce, C. A., Block, R. A., \& Aguinis, H. (2004). Cautionary note on reporting eta-squared values from multifactor ANOVA designs. Educational and Psychological Measurement, 64, 916-924.

Piers, L. S., Soares, M. J., McCormack, L. M., \& O'Dea, K. (1998). Is there evidence for an age-related reduction in metabolic rate? Journal of Applied Physiology, 205, 2196-2204.

Pollock, M. L., Gaesser GA, Butcher JD, Després, J., Dishman, R. K., Franklin, B. A., \& Garber, C. E. (1998). The recommended quantity and quality of exercise for developing and maintaining cardiorespiratory and muscular fitness, and flexibility in healthy adults. Medicine and Science in Sports and Exercise, 30(6), 975991.

Sell, K., Lillie, T., \& Taylor, J. (2008). Energy expenditure during physically interactive video game playing in male college students with different playing experience. Journal of American College Health, 56(5), 505-511.

Tan, B., Aziz, A. R., Chua, K., \& The, K. C. (2002). Aerobic demands of the dance simulation game. International Journal of Sports Medicine, 23(2), 125-129.

Thomas, S., Reading, J., \& Shephard, R. J. (1992). Revision of the physical activity readiness questionnaire (PARQ). Canadian Journal of Sports Science, 17(4), 338-345.

U.S. Department of Health and Human Services. (2008). 2008 Physical Activity Guidelines for Americans. Retrieved from http://www.health.gov/PAGuidelines/guidelines/default.aspx\#toc.

VGCharts Network. American sales charts. (2009). Received from http://www.vgchartz.com/.

Williams, D., Yee, N., \& Caplan, S. E. (2008). Who plays, how much, and why? Debunking the stereotypical gamer profile. Journal of Computer-Mediated Communication, 13(4), 993-1018.

Wilmore, J. (1978). Validity of skinfold and girth assessment for predicting alterations in body composition. Journal of Applied Physiology, 29(3), 313. 
This is an electronic version of an article published in Measurement in Physical Education and Exercise Science, 16(2), 135-150. Measurement in Physical Education and Exercise Science is available online at: www.tandfonline.com. DOI: 10.1080/1091367X.2012.665268

Zhu, W. (2008). Promoting physical activity using technology. Research Digest, 9(3), 1-6. 
Table 1

Nintendo Wii Fit Activities by Category

\begin{tabular}{|c|c|c|c|c|}
\hline Category & Strength & Endurance & Yoga & Balance \\
\hline Activities & $\begin{array}{ll}\text { - } & \text { Single leg extension } \\
\text { - } & \text { Sideways Leg Lift } \\
\text { - } & \text { Single-Arm stand } \\
\text { - } & \text { Torso Twists } \\
\text { - } & \text { Rowing Squat } \\
\text { - } & \text { Single Leg Twist } \\
\text { - } & \text { Lunge } \\
\text { - } & \text { Push-up and Side } \\
\text { - } & \text { Jackknife } \\
\text { - } & \text { Plank } \\
\text { - } & \text { Triceps Extension }\end{array}$ & $\begin{array}{ll}\text { - } & \text { Hula-hoop } \\
\text { - } & \text { Basic Step } \\
\text { - } & \text { Basic Run } \\
\text { - } & \text { Adver Hula Hoop } \\
\text { - } & \text { 2-person Run } \\
\text { - } & \text { Rhythm boxing } \\
\text { - } & \text { Free Step } \\
\text { - } & \text { Free Run }\end{array}$ & $\begin{array}{l}\text { - } \text { Deep breathing } \\
\text { - Half-moon } \\
\text { - } \text { Dance } \\
\text { - } \text { Cobra } \\
\text { - } \text { Bridge } \\
\text { - Spinal Twist } \\
\text { - } \text { Shoulder Stand } \\
\text { - Tree } \\
\text { - Sun salutation } \\
\text { - Standing knee } \\
\text { - Palm tree } \\
\text { - Chair } \\
\text { - Triangle } \\
\text { - Downward Facing Dog }\end{array}$ & $\begin{array}{ll}\text { - } & \text { Soccer heading } \\
\text { - } & \text { Ski slalom } \\
\text { - } & \text { Ski Jump } \\
\text { - } & \text { Table tilt } \\
\text { - } & \text { Tightrope walk } \\
\text { - } & \text { Penguince Bubble Slide } \\
\text { - } & \text { Snowboard } \\
& \text { Slalom } \\
\text { - } & \text { Lotus Focus }\end{array}$ \\
\hline Challenges & $\begin{array}{ll}\text { - } & \text { Push-up } \\
\text { - } & \text { Plank } \\
\text { - } & \text { Jackknife }\end{array}$ & & & \\
\hline
\end{tabular}


Table 2

Randomly Assigned Wii Game Activity Protocol

\begin{tabular}{llll}
\hline Difficulty & Strength Activities & Endurance Activities & Yoga Activities \\
\hline Easy & Lunges (1) & Basic Run Short (2) & Warrior (3) \\
Medium & Single Leg Extensions (4) & Basic Run Long (5) & Chair (6) \\
\hline
\end{tabular}


Table 3

Sample Characteristics

\begin{tabular}{|c|c|c|c|c|c|c|}
\hline \multirow{2}{*}{ Variable } & \multicolumn{2}{|c|}{ Female } & \multicolumn{2}{|c|}{ Male } & \multicolumn{2}{|c|}{ Total } \\
\hline & Mean & SD & Mean & SD & Mean & $\mathrm{SD}$ \\
\hline Age (years) & 22.35 & 1.98 & 21.80 & 2.86 & 22.24 & 2.13 \\
\hline Height (cm) & 166.66 & 5.84 & 176.53 & 7.13 & 168.63 & 7.19 \\
\hline Weight (kg) & 62.55 & 7.41 & 82.34 & 10.69 & 66.50 & 11.30 \\
\hline Body Mass Index $\left(\mathrm{kg} / \mathrm{m}^{2}\right)$ & 22.54 & 2.65 & 26.39 & 2.53 & 23.31 & 3.01 \\
\hline Body Fat \% & 18.75 & 6.40 & 14.57 & 4.48 & 17.92 & 6.22 \\
\hline Lean Body Mass (kg) & 50.43 & 5.49 & 70.08 & 8.18 & 54.36 & 9.97 \\
\hline RMR (mL/Kg/min) & 3.76 & 0.48 & 3.71 & 0.44 & 3.75 & 0.46 \\
\hline $\begin{array}{l}\text { IPAQ Score (MET- } \\
\mathrm{min} / \text { week) }\end{array}$ & 3996 & 2881 & 6426 & 1566 & 4482 & 2822 \\
\hline
\end{tabular}


Table 4

Average $\dot{\mathrm{V}} \mathrm{O}_{2}$ and MET of Wii Activities

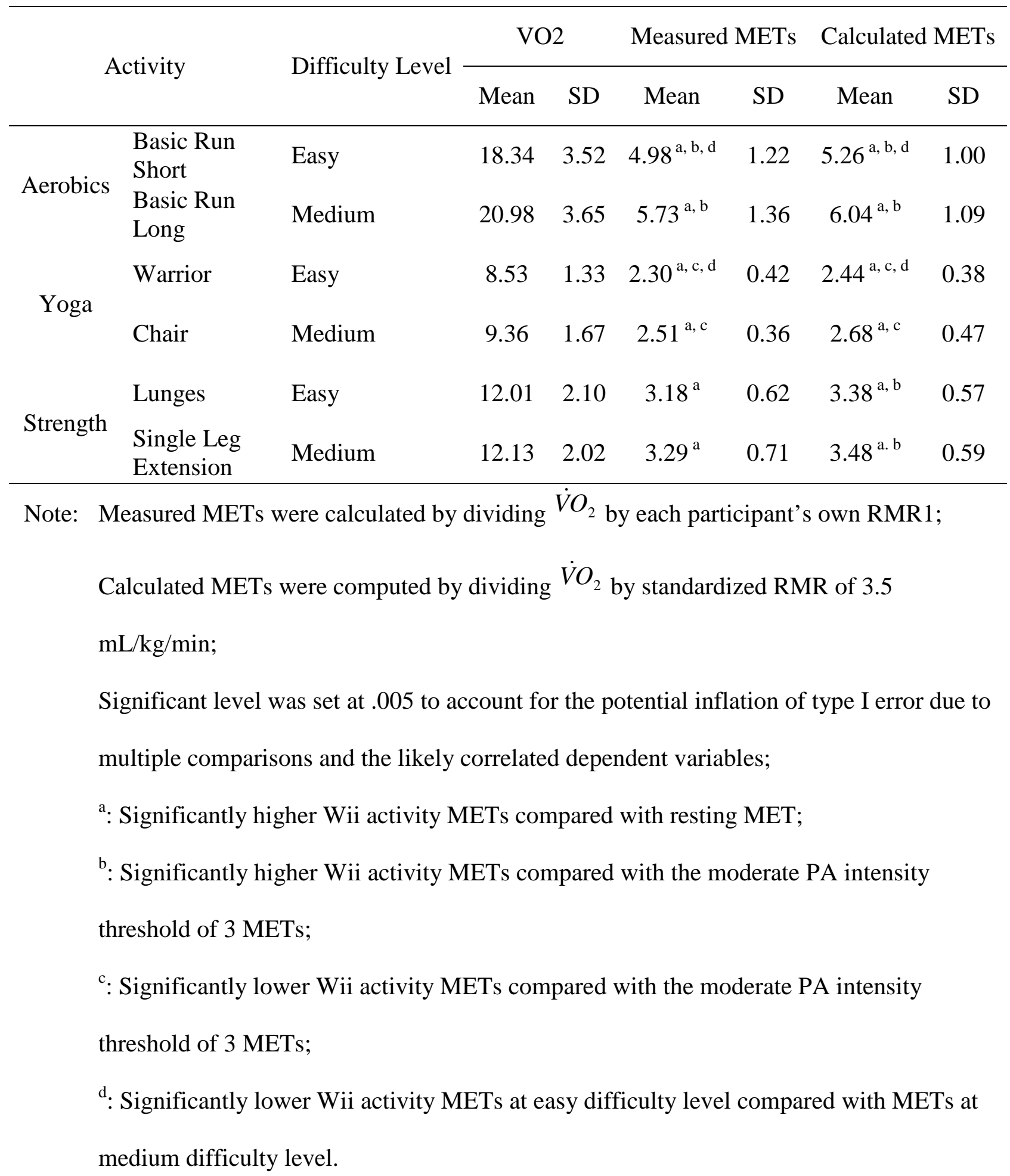

